\title{
A NOTE ON PATHS THROUGH $O$
}

\author{
ROHIT PARIKH
}

ABstract. We show that a hyperarithmetic set can be truth table reduced to a $\Pi_{1}^{1}$-path through $O$ iff it is truth table reducible to some r.e. set.

It is known from results of Feferman and Spector [1] that while there are no $\Sigma_{1}^{1}$-paths through $O$, there do exist $\Pi_{1}^{1}$ such paths. Here by a path is meant a linearly ordered subset $P$ of $O$, closed under $<_{O}$ and having order type $\omega_{1}$.

In this note we prove

THEOREM 1. If $P$ is a $\Pi_{1}^{1}$-path through $O, A$ is hyperarithmetical, and $A$ is truth table reducible to $P$, then the Turing degree of $A$ is at most $\mathbf{0}^{\prime}$.

Thus in a certain sense such paths contain very little "information". It is not known if a hyperarithmetic set of Turing degree greater than $\mathbf{0}^{\prime}$ can be Turing reduced to such a path.

PROOF. We shall actually prove a somewhat stronger fact. If $P$ is a $\Pi_{1}^{1}$-path through $O$ and $A$ is truth table reducible to $P$, then either $A$ is truth table reducible to a proper segment of $P$ (which is necessarily r.e.) or else all of $P$ is used in an essential way, and then one can go backwards and arithmetically decide $P$ from $A$.

So suppose the Turing degree of $A$ is not $\leqq 0^{\prime}$ and $A$ is truth table reducible to such a path $P$. Then there exists a $z$ such that, for all $n, C_{A}(n)=$ $U\left(\mu y T^{P}(z, n, y)\right)$ and moreover, for all $X \subseteq N, U\left(\mu y T^{X}(z, n, y)\right)$ is a total function of $n$. (See [4, p. 143, Theorem XIX].)

Now by (1) there exists a $d \in O^{*}$ and an r.e. ordering $\leq$ such that $P \subseteq P_{1}=\{y \mid y \leq d\}, \leq$ is a linear ordering on $P_{1}$ and $P$ is the maximal well ordered segment of $P_{1}$. (In fact, $\leq$ is that r.e. linear ordering which restricts on $O$ to $<_{O}$.)

Consider pairs $(a, b), a, b \in P_{1}, a<b$, and consider a $z$-computation of $C_{A}(n)$ where: whenever the machine asks, "does $m \in P$ ?" the answer given is "yes" if $m \leq a$, "no" if $m \notin P_{1}$ or $b \leq m$, and no answer is given if neither condition holds. We will say that $(a, b)$ is adequate for $n$ if the correct value of $C_{A}(n)$ is computed in this way.

Received by the editors April 11, 1972.

AMS (MOS) subject classifications (1970). Primary 02F27.

Key words and phrases. Path through $O$, truth table reducible, Turing degree, hyperarithmetic.

(c) American Mathematical Society 1973 
Clearly, there is an $(a, b)$ adequate for a given $n$ and in fact for finitely many $n$. For consider the actual computations of $C_{A}\left(n_{i}\right), i=1, \cdots, k$, from $P$ itself. Let $a=\max$ relative to $\leq$ of all yes answers given and $b=$ min of all no answers, then $(a, b)$ is adequate for $n_{1}, \cdots, n_{k}$. In this case we shall have $a \in P, b \notin P$. However, it is conceivable that $(a, b)$ is adequate for the $n_{i}$ 's and yet both (or neither) of $a, b$ are in $P$. Note that if $(a, b)$ is adequate for $n, a \leq a^{\prime}\left\langle b^{\prime} \leq b\right.$ then $\left(a^{\prime}, b^{\prime}\right)$ is adequate for $n$.

We will say that $(a, b)$ is good, if for all $F \subseteq N, F$ finite, there are $a^{\prime}, b^{\prime}$, $a \leq a^{\prime}\left\langle b^{\prime} \leq b\right.$ such that $\left(a^{\prime}, b^{\prime}\right)$ is adequate for (every element of) $F$. The predicate $G(a, b)((a, b)$ is good) is arithmetical in $A$ and hence hyperarithmetic.

Now we claim that if $G(a, b)$, then $\exists X,\{y \mid y \leq a\} \subseteq X \subseteq\{y \mid y \leq b\}$ and $C_{A}(n) \equiv U\left(\mu y T^{X}(z, n, y)\right)$.

For note that if $a<c<b$ and $G(a, b)$ then either $G(a, c)$ or $G(c, b)$. Otherwise there are finite sets $F_{1}, F_{2}$ such that no pair $\left(a^{\prime}, b^{\prime}\right)$ contained in $(a, c)$ is adequate for $F_{1}$, and no pair $\left(a^{\prime}, b^{\prime}\right)$ contained in $(c, b)$ is adequate for $F_{2}$. But then no pair $\left(a^{\prime}, b^{\prime}\right)$ contained in $(a, b)$ is adequate for $F_{1} \cup F_{2}$ contradicting the goodness of $(a, b)$. (Either $\left(a^{\prime}, b^{\prime}\right)$ is contained in $(a, c)$ or in $(c, b)$ or else $\left(a^{\prime}, c\right)$ is adequate for $F_{1}$.) by:

Now given $G(a, b)$, let $c_{1}, c_{2}, \cdots$ be an enumeration of $P_{1}$. Define $\left(a_{i}, b_{i}\right)$

$$
\begin{array}{rlrl}
\left(a_{0}, b_{0}\right) & =(a, b), & \\
\left(a_{n+1}, b_{n+1}\right) & =\left(a_{n}, b_{n}\right) & & \text { unless } a_{n}<c_{n+1}<b_{n}, \\
& =\left(a_{n}, c_{n+1}\right) & \text { if } a_{n}<c_{n+1}<b_{\imath} \text { and } G\left(a_{n}, c_{n+1}\right), \\
& =\left(c_{n+1}, b_{n}\right) & & \text { otherwise. }
\end{array}
$$

Then $G\left(a_{n}, b_{n}\right)$ holds for all $n$ and no $x$ can satisfy $a_{n}<x<b_{n}$ for all $n$. Let $X$ be the set $\left\{x \mid(\exists n)\left(x \leq a_{n}\right)\right\}$. Claim $U\left(\mu y T^{X}(z, n, y)\right)$ always equals $C_{A}(n)$.

Otherwise (by choice of $z$ ) there is a $p$ such that $U\left(\mu y T^{X}(z, p, y)\right)$ is defined and unequal to $C_{A}(p)$. Consider $a$, the maximum (in $\leq$ ) of all the "yes" answers during this computation from $X$. Similarly $b$ is the minimum of all "no" answers for elements of $P_{1}$. Then there is an $n$ such that $a \leq a_{n}<b_{n} \leq b$. But clearly $\left(a_{n}, b_{n}\right)$ cannot be good since no pair $\left(a^{\prime}, b^{\prime}\right)$ contained in $(a, b)$ can be adequate for $\{p\}$.

Thus $G(a, b)$ implies $b \notin P$. Otherwise an $X$ such as above would be a proper segment of $P$, and hence r.e. But clearly $b \in P_{1}-P$ implies that for all $a \in P, G(a, b)$. Hence we get

$$
x \in P \leftrightarrow x \in P_{1} \wedge \neg(\exists a)(G(a, x)),
$$

a contradiction since $P$ is not hyperarithmetic. Q.E.D. 
It is fairly straightforward to show that the lower bound on the degree of $A$ is best possible.

THEOREM 2. ${ }^{1} \quad$ There is a $\Pi_{1}^{1}$-path $P$ through $O$ such that the set $A=$ $\{n \mid(\exists y) T(n, n, y)\}$ is $1-1$ reducible to $P$.

Proof. Define an ordering $R$ on $N$ by

$$
R(x, z) \leftrightarrow(\exists y)(T(x, x, y) \wedge(\forall u \leqq y)(\neg T(z, z, u)) .
$$

This is a partial ordering, the elements in $A$ are a sequence of type $\omega$ and the elements not in $A$ are a set of incomparables above this sequence. Now apply the construction of $[3$, p. 45] to this ordering. We get a function $g$ from $N$ to $O$ such that $g[N-A]$ is a set of incomparables and if $x \in A, y \in N-A$ then $g(x)<o g(y)$. Take any $x_{0} \in N-A$ and pick $d \in O^{*}$ such that $g\left(x_{0}\right)<_{O} d$. The path $P$ corresponding to $d$ has the required property.

REMARK. Note that $A$ is in fact $1-1$ reduced to a proper segment $S$ of $P$. $P-S$ plays no role, since $g[A] \subseteq S$ and $g[N-A] \subseteq N-P$.

\section{REFERENCES}

1. S. Feferman and C. Spector, Incompleteness along paths in progressions of theories, J. Sỳmbolic Logic 27 (1962), 383-390. MR 30 \#3012.

2. G. Kreisel, Which number theoretic problems can be solved in recursive progressions on $\Pi_{1}^{1}$-paths through $O$ ?, J. Symbolic Logic 37 (1972), 311-334.

3. G. Kreisel, J. Shoenfield and H. Wang, Number theoretic concepts and recursive well-orderings, Arch. Math. Logik Grundlagenforsch 5 (1960), 42-64. MR 22 \#6709.

4. H. Rogers, Jr., Theory of recursive functions and effective computability, McGrawHill, New York, 1967. MR 37 \#61.

Department of Mathematics, Boston University, Boston, Massachusetts 02215

${ }^{1}$ This result was also found, independently and simultaneously by C. Jockusch. 